\title{
Psicología social y Metodologías Cualitativas: La investigación como herramienta de construcción social
}

\author{
Introducción. \\ Sobre el continuo entre investigación y realidad psicosocial: la metodología como \\ mediación
}

\author{
Dr. Jorge Castillo-Sepúlveda \\ Escuela de Psicología \\ Universidad de Santiago de Chile
}

La psicología social es comúnmente identificada como un campo disciplinar que aborda cómo los procesos sociales y culturales influyen en la constitución de la subjetividad y, a la inversa, cómo las personas contribuyen a la formulación de fenómenos o formaciones sociales más amplias, que les trascienden, estableciendo modos de relación que se perpetúan en el tiempo y el espacio. Esta definición, si bien amplia, supone el anclaje de un ámbito ontológico particular, que podría esbozarse de la siguiente manera: la psicología social atiende a las relaciones entre personas y sus efectos, considerando cómo estas se dan y producen en un contexto histórico, cultural y lingüístico. Así, más allá de considerar cómo una persona se desarrolla a sí misma y construye su propia historia, la psicología social dirá que esta es producto de una serie de procesos y relaciones complejas en que participan la cultura, el lenguaje -como expresión específica de cultura-y la sociedad en su decurso histórico. Se trata de una ontología que asume la historicidad como componente base de una serie de procesos de afección recíproca entre componentes de índole sociocultural (véase Tirado, 1996). Ahora bien, como disciplina y formación cultural, la misma psicología social se entrelaza a este tipo de procesos y se constituye a sí misma como un objeto social (Ibáñez, 1993). En tal sentido, quizás como en cualquier otra disciplina, existen vínculos de permeabilidad entre las transformaciones sociales y los aspectos prácticos y científicos de la psicología social; así, en tanto se modifican los modos de relacionarnos al emerger nuevos mediadores sociales, nuevas tecnologías y contextos socio-económicos y políticos, la psicología social no sólo varía en sus objetos, sino también requiere de modos diversos para aproximarnos a esta realidad, dar cuenta de ella y, en diversos sentidos, construirla mediante estas mismas actividades de generación de conocimiento (Ibáñez, 1993; Potter, 1998).

El reconocimiento de la función constructiva del conocimiento no es propio de la psicología social, y se inserta en una extensa tradición de debates filosóficos respecto al papel de quienes conocemos y aquello que es conocido, sus distancias, límites o posibilidades de afección recíproca (véase, por ejemplo, Ibáñez, 1998). Sin embargo, es posible reconocer un resurgimiento especial de este debate en el ámbito disciplinar de la psicología social a partir de la década de 1960, en que se instaló una serie de críticas a los modelos epistemológicos y, junto a ello, metodológicos de la psicología social dominante en la época; en particular a la psicología social de carácter experimental o llevada a cabo de modo exclusivo en laboratorios. En este contexto, dos artículos publicados en el Journal of Experimental Social Psychology, en el año 1967, por Kenneth Ring y William McGuire, sirvieron de base para una serie de intercambios en instancias de difusión científica respecto al rol, posición y función de la psicología social en los escenarios políticos y sociales de la época. Además, abordaron lo vergonzoso y trivial que resultaban, en variadas ocasiones, los objetivos, aproximaciones y aplicaciones de la psicología social. Estas temáticas, en lugar de socavarse o haber encontrado una resolución a partir de los distintos debates e instancias de diálogo 
generados durante los últimos, casi, cincuenta años, han promovido la instalación de múltiples versiones de la psicología social, empleando supuestos y desarrollando metodologías diversas, que derivan en la promulgación, por tanto, de variadas realidades académicas y sociales (Prioli Cordeiro, 2012). La cuestión del enlace entre disciplina, metodología y realidad, por tanto, no se reduce a una pura cuestión técnica relativa a la confiabilidad o validez que habiliten determinadas estrategias, sino, mediante estas estrategias, a la producción misma de los contextos o situaciones a las cuales se atiende y que se constituyen en material de debates políticos y sociales (véase, por ejemplo, Mol, 1999, 2002).

Existe, entonces, "una intensa ligazón, por no decir una 'no-separabilidad' entre la producción de conocimientos por una parte, y la construcción de los objetos de conocimiento por otra parte" (Ibáñez, 1993, p. 30):

Sin caer en una epistemología idealista, para la cual la "realidad" es equivalente a lo que se cree que es, es preciso reconocer que la psicología social no se ciñe a informarnos sobre las características de la intersubjetividad contemporánea, sino que contribuye a constituir esas características en el propio proceso de su investigación. (p. 30)

La fórmula de Ibáñez (1993) respecto al bucle recursivo entre construcción de conocimiento y construcción de objetos de conocimiento deriva en un supuesto clave para la psicología social, o por lo menos para su vertiente más crítica: "los conocimientos elaborados por la psicología social pueden incidir sobre las realidades sociales que pretende conocer" (p. 30) y ello hace imperioso generar un proceso reflexivo respecto a los procedimientos con que se investigue y los aspectos éticos y políticos implicados (Ibáñez, 1993). La naturaleza y sensibilidad de los objetos a este conocimiento quedan circunscritas por las posibilidades de ser mediatizados por las significados promovidos por esta disciplina; lo subjetivo, social, cultural, histórico e, inclusive, lo material, son aspectos susceptibles de ser significados y, por lo tanto, sensibles a los efectos de las teorías y las actividades de investigación psicosociales (Flores-Pons, Íñiguez \& Martínez-Guzmán, 2013, Mayo; Tirado, 2011, en comunicación personal).

$\mathrm{Al}$ respecto, la importancia de la metodología no atañe solo al trazado del recorrido ${ }^{1}$ a la delimitación

\footnotetext{
La raíz de metodología proviene del griego metá, que significa "fuera o más allá" y odós, que refiere a camino o viaje. Un método remite a algo que está "más allá del viaje o del camino" (Divry, 1969)
}

de una estrategia particular para llevar a cabo una investigación; lo metodológico es, ante todo, un aspecto procedimental que contribuirá a dar cuenta de unos u otros aspectos del objeto de investigación, de unos rasgos por sobre otros y, en tal medida, contribuirá a la construcción del aspecto psicosocial que se investiga (Ramos, 2012). Si las teorías y conceptos pueden ser comprendidos como una caja de herramientas para pensar y referirnos a lo psicosocial (Garay, Íñiguez \& Martínez, 2002), la metodología -siguiendo esta metáfora- remite a cómo estas herramientas entran en contacto con sus objetos, les perfilan y adquieren una forma a partir de una masa uniforme de pura socialidad. Remite a una mediación importantísima en el trabajo de investigación y, en este sentido, consiste en un aspecto que definirá en gran medida el objeto de nuestro estudio, en tanto permitirá describir ciertas cualidades o determinadas intensidades del aspecto psicosocial que se aborde.

Aquí, la noción de mediación es empleada en un sentido particular. Con este término nos referimos a la labor eminentemente transformadora de una metodología respecto a los propósitos de una investigación: un andamiaje entre teoría y objeto. Una mediación es una acción, pero una tal que participa de otras acciones y las transforma. No consiste en una intermediación en el sentido de servir de canal para la transferencia entre dominios distintos que pretenden entrar en contacto, sino es aquello que habilita-hablamos teóricamente- este contacto, esto es "un acontecimiento que no es del todo una causa, ni del todo una consecuencia, ni completamente un medio o un fin" (Latour, 1999, citado en Tirado, 2001, p. 277; en referencia al concepto de mediación, no a su uso en referencia a la metodología). Así, en tanto habilita un contacto, establece sus condiciones y las categorías por las cuales éste será inscrito en los diversos actores. En otros términos, no son sólo las condiciones para afrontar un camino, sino que es la trayectoria en sí misma.

Los últimos decenios han visto cómo desde una predominancia de estrategias cuantitativas para la aproximación a los fenómenos psicosociales (principalmente a través de escalas normalizadas, y tratamiento estadístico de información recogida mediante técnicas ad hoc), han adquirido difusión en ámbitos académicos de distinta naturaleza metodologías de orientación cualitativa (que atienden, según comúnmente se define, a los aspectos de significado o sentido; las "cualidades" de los fenómenos psicosociales). Si bien puede reconocerse que ambas aproximaciones se han enfrentado en una suerte de cruzada epistemológica, pretendiendo estratégicamente dar cuenta de la superioridad práctica o conceptual de una u otra, consideramos que este giro 
metodológico obedece no a una victoria -transitoriaentre estos debates, sino a una cuestión de un orden distinto. Se trata del reconocimiento -o adopción- de una lógica en que los dispositivos de medición se entraman en razones que son predominantemente discursivas. De este modo, si hay fenómenos que son esencialmente cuantificables es porque "los dispositivos de intercambio [categorías] incluyen dispositivos de cuantificación o medida -dispositivos de valoración-" (Ibáñez, 1985, p.107), es decir, se han elaborado estrategias para medir y para narrar diferenciadamente, sin que ello de cuenta de modo necesario del fenómeno que atañe. No obstante, cualquiera sea el dispositivo, este depende de y es fabricado en entramados de referencia o significación $\mathrm{y}$, por lo tanto, en discursos que los sustentan. Creemos que este giro -ni total, ni propiamente extensivo, mas enunciado (véase Íñiguez, 2006) - obedece a la connotación que ha adquirido lo discursivo como un elemento que promueve ciertos modos de socialidad, y que genera efectos particulares en sus desplazamientos.

Es por el papel fundamental de lo metodológico en la investigación psicosocial, y en particular en las reflexiones que son parte de las metodologías cualitativas, que en esta primera sección especial de la Revista Summa Psicológica hemos querido recoger cuatro investigaciones que ensayan estrategias metodológicas específicas en este ámbito. Si bien cada una atiende a un aspecto diferencial de las realidades sociales y culturales a las que refieren -también distintas-, el aspecto que las aglutina remite al abordaje reflexivo respecto de la metodología empleada y, junto a ello, del papel de el(la) investigador(a), de los conceptos y teorías implicados, y de la función investigadora en sí misma en relación a los procesos psicosociales.

En relación a lo anterior, y atendiendo a una serie de fenómenos de difusión cultural mediante estrategias mediáticas soportadas por el empleo masivo de Internet, Rodrigo Bassaletti-Contreras aborda los procesos y posibilidades de integración en el contexto sociocultural chileno del anglicismo bullying, atendiendo especialmente a las condiciones de los contextos socioeducativos nacionales inscritos en las políticas públicas. Con a ello, establece las problemáticas asociadas a la incorporación de significantes construidos en realidades políticas y culturales extranjeras, en los entramados lingüísticos y prácticos de los contextos chilenos, realizando un análisis desde una óptica postcolonial, en el sentido de la reproducción y objetivación local de perspectivas sobre la violencia formulada en países nórdicos. Para ello, lleva a cabo un análisis del discurso del material visual y textual presente en diversos videos producidos en Chile sobre "el” bullying, difundidos públicamente en Internet. Entre las múltiples cualidades de esta investigación, destacan la integración en una metodología de estrategias y técnicas diversas, junto al talante reflexivo presente en la totalidad del proceso de investigación respecto, por ejemplo, a las fuentes y trayectoria histórica del constructo en sí mismo, y las funciones políticas implicadas en la construcción y difusión de conceptualizaciones científicas.

En esta misma línea reflexiva, Karina FulladosaLeal, despliega en su investigación una metodología que materializa o convierte en textualidad los procesos de interacción e intercambio entre investigador(a) e investigado(a). A través del empleo de producciones narrativas, Fulladosa-Leal expone en su artículo los relatos de experiencias, reflexiones propias de la investigadora y elaboraciones teóricas al respecto, leídas y transformadas por las investigadas, sobre el trabajo de reproducción social y de cuidado en circunstancias potenciales de vulneración de derechos, situación ampliamente presente pero invisibilizada en sociedades en que se presentan flujos migratorios periódicos. La exposición de la investigadora es constante en todo el proceso investigativo, así como una propuesta política al respecto.

En un ámbito diverso, y desde una perspectiva distinta, Daniela Osorio-Cabrera analiza en su artículo las posibilidades y dificultades que enfrenta un colectivo que participa en la red de Economía Solidaria en Cataluña, para encarnar su propio discurso relativo a la transformación de las relaciones de género respecto al trabajo. En este sentido, analiza los modos de reproducción de discursos que explícitamente son resistidos por dicho colectivo, estableciendo formas en que este opera y, a la vez, aspectos no visibilizados en los cuales esta variación o resistencia entra en acción. Para ello, lleva a cabo un análisis de contenido, estrategia que, si bien utilizada comúnmente como método cualitativo, es asociada a una serie de consideraciones que dan a la investigación un estatuto que se desplaza de lo eminentemente descriptivo, para, junto a ello, esbozar un trabajo propositivo respecto a la connotación de procesos que participan del proyecto transformador del grupo.

Y, en el ámbito de los desarrollos investigativos en torno a la noción de resistencia, María Teresa Ramírez presenta en la experiencia de una comunidad de $o k u$ pación rural española, los mecanismos discursivos mediante los cuales un colectivo pretende establecer variaciones en relación a los modos de vida dominante, exponiendo, a su vez, las dificultades presentadas en este proceso. Desarrollando una estrategia metodológica que ha denominado como grupos reflexivos, devela 
a partir del intercambio de vivencias y saberes, incluso de la misma investigadora, los aspectos que limitan que el proyecto del movimiento de okupación rural sea actualizado de modo que establezca diferenciaciones puras respecto a la hegemonía cultural. En este sentido, la autora esclarece la función particular que un grupo de minoría cultural puede jugar en los procesos de transformación social, no en el sentido propio de oposición a la cultura, sino en el de aceleración de movimientos sociopolíticos hegemónicos.

Consideramos que los cuatro artículos que componen esta sección especial, si bien no representan la labor extensiva de la psicología social y las metodologías cualitativas, pueden dar cuenta de la función mediadora de los aspectos metodológicos y productivos de la investigación psicosocial. Junto a ello, la figura de el(la) investigador(a) se expone no sólo como un espacio de articulación de elementos teóricos y relativos al contenido o efecto de los discursos de las personas que participan en las investigaciones, sino como un componente también mediado por los diversos dispositivos de los que da cuenta. De tal modo, un investigador no sólo emplea ciertos procesos para exponer sus objetos, sino también es empleado por estos, en el sentido de brindar la oportunidad de su actualización y desarrollo. En cierta forma, investigador(a) y metodología quedan prehendidos, es decir, inscritos en una relacionalidad que define su mutua identidad.

\section{Referencias}

Divry G. (Ed.) (1969). Divry's new English-Greek and Greek-English handy dictionary. Nueva York: D.C. Divry.

Flores-Pons, G., Íñiguez, L. \& Martínez-Guzmán, A. (2013, mayo). Discourse, materiality and performativity: Reflections on the illocutionary force of objects. 15th Meeting of the International
Society of Theoretical Psychology: Dialogue and debate in the making of theoretical psychology. Pontificia Universidad Católica de Chile, Santiago, Chile.

Garay, A., Íñiguez,L. \& Martínez, L.M. (2002). Perspectivas críticas en Psicología social: herramientas para la construcción de nuevas psicologías sociales. Boletín de psicología, 72, 57-78.

Ibáñez, J. (1985). Las medidas de la sociedad. Reis: Revista Española de Investigaciones Sociológicas, 29, 85-127.

Ibáñez, T. (1993). La dimensión política de la psicología social. Revista Latinoamericana de Psicología, 25(1), 19-34.

Ibáñez, T. (1998). Cómo ser relativista y no morir en el intento. Revista de la Universidad de Guadalajara, 11, 58-65.

Íñiguez, L. (Ed.) (2006). Manual de análisis del discurso. Barcelona: Editorial UOC.

McGuire, William (1967). Some empendings reorientations in social Psychology. Journal of Experimental Social Psychology, 3(2), 124-139.

Mol, A. (1999). Ontological politics. A word and some questions. En J. Law y J. Hassard (Eds.), Actor Network Theory and after (pp.74-89). Oxford: Blackwell Publishing.

Mol, A. (2002). The body multiple: ontology in medical practice. Durham, North Carolina: Duke University Press.

Potter, J. (1998). La representación de la realidad. Discurso, retórica y construcción social. Barcelona: Paidós.

Prioli Cordeiro, M. (2012). Psicologia Social no Brasil: multiplicidade, performatividade e controversias. Tesis para optar al grado académico de Doctor en Psicología Social, Pontifícia Universidade Católica de São Paulo, PUC-SP, São Paulo, Brasil.

Ramos, C. (2012). Investigación científica y performatividad social: el caso de la PNUD en Chile. En T. Ariztía (Ed.), Produciendo lo social: usos de las ciencias sociales en el Chile reciente (pp. 219-252). Santiago, Chile: Ediciones Universidad Diego Portales.

Ring, K. (1967). Experimental Social Psychology: Some sober questions about some frivolous values. Journal of Experimental Social Psychology, 3(2), 113-123.

Tirado, F. (1996). El cómo y el por qué de la psicología social. Introducción. En T. Ibáñez (Coord.), Introducción a la psicología social (pp. 53-58). Barcelona, España: Editorial UOC.

Tirado, F. (2001). Los objetos y el acontecimiento: teoría de la socialidad mínima. Tesis para optar al grado académico de Doctor, Unitat de Psicologia Social, Departament de Psicologia de la Salut i de Psicologia Social, Facultat de Psicologia, Universitat Autònoma de Barcelona. 